\title{
The proper motion and energy distribution of the isolated neutron star RX J0720.4-3125
}

\author{
C. Motch ${ }^{1, \star}$, V. E. Zavlin ${ }^{2}$, and F. Haberl ${ }^{2}$ \\ 1 Observatoire Astronomique, UA 1280 CNRS, 11 rue de l'Université, 67000 Strasbourg, France \\ 2 Max-Planck-Institut für extraterrestrische Physik, 85740 Garching bei München, Germany
}

Received 12 March 2003 / Accepted 12 May 2003

\begin{abstract}
ESO 4 m class telescope and VLT deep imaging of the isolated neutron star RX J0720.4-3125 reveals a proper motion of $\mu=97 \pm 12 \mathrm{mas} / \mathrm{yr}$ and a blue $U-B$ color index. We show that a neutron star atmosphere model modified to account for a limited amount of hydrogen on the star's surface can well represent both the optical and X-ray data without invoking any additional thermal component. The large proper motion almost completely excludes the possibility that accretion from the interstellar medium is the powering mechanism of the X-ray emission. It also implies that the proposed spin down is entirely due to magnetic dipole losses. RX J0720.4-3125 is thus very likely a middle aged cooling neutron star. Its overall properties are quite similar to some of the long period radio pulsars recently discovered, giving further support to the idea that RX J0720.4-3125 may be a pulsar whose narrow radio beam does not cross the Earth.
\end{abstract}

Key words. stars: newton - X-rays: individual: RX J0720.4-3125

\section{Introduction}

ROSAT has identified a small group of hot and radio-quiet isolated neutron stars (INSs, see reviews by Motch 2001; Treves et al. 2001; Haberl 2003). None of these objects seems associated with a SNR and low interstellar absorption towards them indicates distances of the order of a few hundred parsecs at most. Grating X-ray spectra of the two brightest members, RX J0720.4-3125 (Paerels et al. 2001) and RX J1856.5-3754 (Burwitz et al. 2001; Drake et al. 2002), do not significantly depart from pure blackbody energy distributions. Inferred blackbody temperatures range between 50 and $100 \mathrm{eV}$. Soft X-ray pulsations with periods around $10 \mathrm{~s}$ are now detected in a majority of sources although some period candidates still require confirmation. Optical counterparts are very faint objects whose brightness seems to be mostly due to thermal emission from the neutron star surface (see Pavlov et al. 2002 for a recent review). These INSs undergo little, if any, magnetospheric activity and are thus prime targets to measure fundamental parameters through modeling the neutron star thermal emission and to constrain the debated equations of state.

The evolutionary status of these objects is still unclear. Some of the $10^{8}$ to $10^{9}$ INSs born in the Galaxy could have

Send offprint requests to: C. Motch, e-mail: motch@newb6.u-strasbg.fr

* Based on observations collected at the European Southern Observatory, La Silla and Paranal, Chile (ESO Programmes 58.D-0596, 62.H-0706, 64.H-0156(A), 66.D-0286(A) and 70.D-0515(A) space velocities and rotation periods slow enough to accrete from relatively dense phases of the interstellar medium and be re-heated to temperatures similar to those observed (see e.g. Blaes \& Madau 1993). The fraction of old INSs detectable in the X-ray is of the order of several percent but strongly depends on the assumed velocity distribution (Madau \& Blaes 1994). This hypothesis currently faces two main difficulties. First, the space density of hot radio-quiet INSs is much lower than predicted by the accretion models (Treves et al. 2001). Second, the rotation periods, although relatively long for neutron star standards, are still too short to allow accretion to take place in average interstellar medium conditions unless the magnetic fields of INSs have significantly decreased by about two orders of magnitudes over their life time (see e.g. Wang 1997).

Alternatively, these INSs could be young cooling objects. Long rotation periods are observed in a few radio pulsars as well as in AXPs, showing that under certain circumstances (efficient braking or birth with a low angular momentum), relatively young neutron stars can rotate slowly.

Optical observations can efficiently help to discriminate between the two hypotheses, re-heating by accretion from the insterstellar medium or relatively young cooling objects. For some of the objects, proper motions can be measured which, assuming Bondi-Hoyle accretion, may rule out the accreting scenario. Additionally, the optical to X-ray flux ratio is sensitive to the chemical composition of the neutron star surface (Pavlov et al. 1996) and may therefore reveal the presence of newly accreted material from the interstellar medium or from a fossil disc. 
Table 1. Optical data.

\begin{tabular}{llcccc}
\hline \hline Date & $\begin{array}{l}\text { Instrument } \\
\text { telescope }\end{array}$ & Band & $\begin{array}{c}\text { Exp. } \\
(\mathrm{ks})\end{array}$ & $\begin{array}{c}\text { Pixel } \\
\left({ }^{\prime \prime}\right)\end{array}$ & $\begin{array}{c}\text { Seeing } \\
\left({ }^{\prime \prime}\right.\end{array}$ \\
\hline 7-11 Feb. 1997 & SUSI/NTT & $U$ & 7.2 & $0.13^{\prime \prime}$ & \\
7-11 Feb. 1997 & SUSI/NTT & $B$ & 5.4 & $0.13^{\prime \prime}$ & \\
10-11 Mar. 1997 & SUSI/NTT & $U$ & 3.6 & $0.13^{\prime \prime}$ & \\
29 Mar. 1997 & SUSI/NTT & $B$ & 1.8 & $0.13^{\prime \prime}$ & \\
29 Mar. 1997 & SUSI/NTT & $U$ & 1.8 & $0.13^{\prime \prime}$ & \\
4 Apr. 1997 & SUSI/NTT & $B$ & 1.8 & $0.13^{\prime \prime}$ & \\
\hline Sum $B$ 1997 & SUSI/NTT & $B$ & 9.0 & $0.13^{\prime \prime}$ & $0.91^{\prime \prime}$ \\
Sum $U$ 1997 & SUSI/NTT & $U$ & 12.6 & $0.13^{\prime \prime}$ & $0.97^{\prime \prime}$ \\
\hline 15 Jan. 1999 & SUSI2/NTT & $B$ & 12.0 & $0.157^{\prime \prime}$ & $0.80^{\prime \prime}$ \\
15 Jan. 1999 & SUSI2/NTT & $R$ & 6.0 & $0.157^{\prime \prime}$ & $0.82^{\prime \prime}$ \\
\hline 7-8 Jan. 2000 & EFOSC2/ESO-3.6 m & $B$ & 17.4 & $0.314^{\prime \prime}$ & $0.94^{\prime \prime}$ \\
7-8 Jan. 2000 & EFOSC2/ESO-3.6 m & $U$ & 26.1 & $0.314^{\prime \prime}$ & $0.94^{\prime \prime}$ \\
\hline 20-23 Dec. 2000 & FORS1/VLT-UT1 & $B$ & 24.1 & $0.10^{\prime \prime}$ & $0.60^{\prime \prime}$ \\
\hline 28 Dec. 2002-2 Jan. 2003 & FORS1/VLT-UT1 & $B$ & 24.8 & $0.10^{\prime \prime}$ & $0.5-1.2^{\prime \prime}$ \\
\hline
\end{tabular}

In both the optical and X-ray band, RX J0720.4-3125 is the second brightest INS among those discovered with ROSAT. This object exhibits pulsations at a period of $8.39 \mathrm{~s}$ (Haberl et al. 1997) with $|\dot{P}|<3.6 \times 10^{-13} \mathrm{~s} \mathrm{~s}^{-1}$ (Kaplan et al. 2002). The X-ray spectrum is blackbody-like with $k T^{\infty} \simeq 86 \mathrm{eV}$ (as given by the ROSAT data). ESO-NTT and Keck observations have led to the optical identification of RX J0720.4-3125 with a faint stellar-like object (Motch \& Haberl 1998; Kulkarni \& van Kerkwijk 1998).We report here on new 4 m-class telescope and ESO-VLT observations of RX J0720.4-3125 which reveal its proper motion, show its blue UV continuum and provide constraints on the nature of its thermal emission. HST observations of RX J0720.4-3125 (Kaplan et al. 2003) confirm the steep optical to UV energy distribution of the star.

\section{Optical observations}

Optical imaging was obtained over five ESO periods spanning the time interval from February 1997 till January 2003. The log of observations is given in Table 1. Data collected in 1997 have been already presented and discussed in Motch \& Haberl (1998). In January 1999 we used the ESO-NTT and the SUSI2 imager equipped with EEV44-80 CCDs \#45 and \#46 (2048 × 4096 pixels). The January 2000 run was carried out at the ESO $3.6 \mathrm{~m}$ using EFOSC2 and the LORAL $2048^{2}$ CCD \#40. At UT1 we used in both cases FORS1 and a TK $2048^{2}$ CCD. Individual exposures of $\sim 10 \mathrm{~min}$ were obtained in 1997, 1999, 2000 and 2002-2003 for the $B$ and $R$ bands while the $U$ band 2000 images were $15 \mathrm{~min}$ long. In January 1999 and 2000, the $U$ and $B$ filters were alternated and in all instances the telescope was moved by a few arcseconds in different directions between two exposures. Raw images were corrected for bias using overscan regions or mean bias frames and corrected for flat-field using dithered sky images obtained at dawn. Individual corrected images were then moved to a common frame using a set of several nonsaturated bright stars and then stacked together applying a statistical criterion in order to reject cosmic-ray impacts. Because of the excellent seeing prevailing during ESO-VLT observations, the geometrical distortion effects of the FORS camera (up to $0.2^{\prime \prime}$ on the edge of the field) combined with dithering slightly deteriorated the quality of the stacked images. Distortion effects were thus corrected before stacking using the midas rectify image task and parameters taken from the FORS users Manual (Issue 1.5). After correction, the positional errors of reference stars dropped from 30 mas to 5 mas and the $F W H M$ image size sharpened by 0.1 to $0.05^{\prime \prime}$.

\section{Optical photometry}

Figure 1 shows the deep $B$ image obtained by summing the $3.4 \mathrm{~h}$ FORS1 observation in 2002-2003 of best image quality resulting in an average $F W H M$ seeing of $0.56^{\prime \prime}$. The optical counterpart of RX J0720.4-3125 (object "X1" from Motch \& Haberl 1998 or "X" in Kulkarni \& van Kerkwijk 1998) is clearly detected, thanks to the excellent images and VLT throughput. Our relatively deep $U$ exposure obtained by summing the $7.2 \mathrm{~h}$ long observation in January 2000 collected at the ESO-3.6 m (see Fig. 2) confirms the strong UV excess of the counterpart seen by Kaplan et al. (2003). Object "X2" in Motch \& Haberl (1998) does not appear exactly at the same position in the 1997 image as in the Keck observation of Kulkarni \& van Kerkwijk (1998) or as in our 1999, 2000 January and VLT images. We believe that a low amplitude unfiltered sky background fluctuation could be the origin of its displacement. The revised position of "X2" outside of the ROSAT error box and its lower UV flux excludes it as a possible counterpart of RX J0720.4-3125 (Kulkarni \& van Kerkwijk 1998). Our $R$ image in January 1999 is not deep enough to detect the counterpart.

Absolute photometric calibration was obtained in the course of the January 2000 run. Standard stars in the fields of PG 0231+051, PG 0942-029 and PG 1047+003 


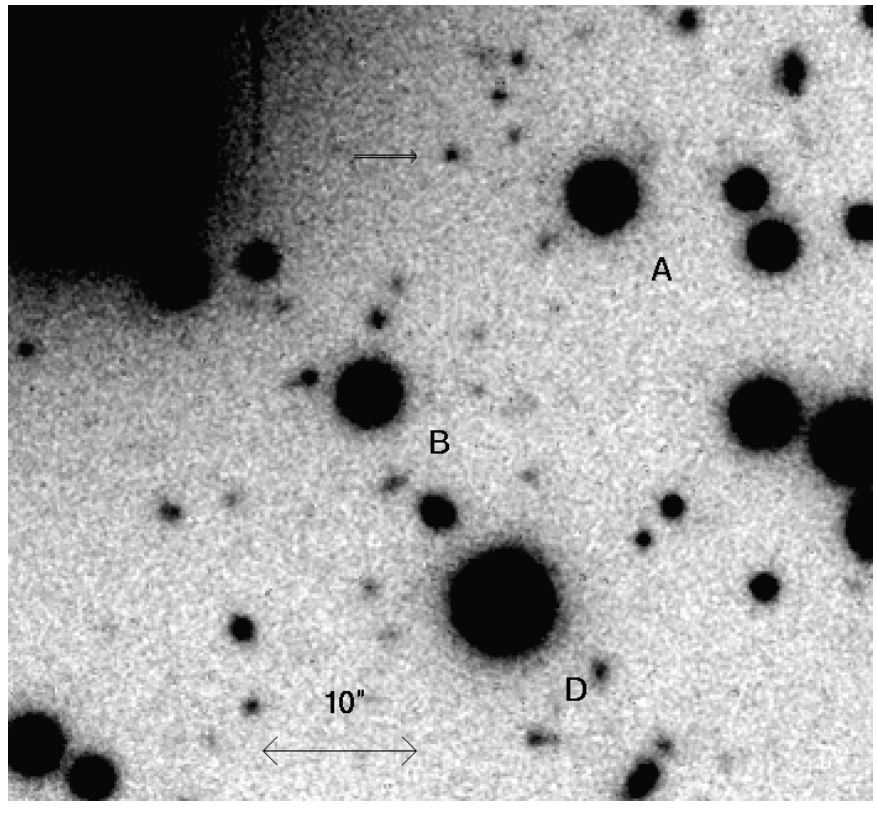

Fig. 1. Sum of best seeing $B$ band images obtained with FORS1 on UT1 in December 2002 and January 2003. Positions of the photometric reference stars are shown. North is to the top and East is to the left. The arrow marks the position of RX J0720.4-3125.

Table 2. Magnitudes of field reference stars ( $1 \sigma$ errors).

\begin{tabular}{ccc}
\hline \hline Star & $U$ & $B$ \\
\hline A & $20.585 \pm 0.014$ & $20.542 \pm 0.009$ \\
B & $21.011 \pm 0.015$ & $20.841 \pm 0.009$ \\
D & $18.770 \pm 0.014$ & $18.766 \pm 0.008$ \\
\hline
\end{tabular}

(Landolt 1992) were used to derive $U$ and $B$ magnitudes of three comparison stars named A, B and D located close to RX J0720.4-3125. Stars A and B are the same as in Haberl et al. (1997). Their magnitudes are listed in Table 2 and their positions are shown in Fig. 1. The sky background close to RX J0720.4-3125 is somewhat contaminated by stray light from a nearby group of relatively bright stars. Because of its equatorial mounting images obtained at the ESO $3.6 \mathrm{~m}$ further suffer from fixed diffraction spikes caused by the secondary spider. Following Kulkarni \& van Kerkwijk (1998), we found that the most accurate photometric measurements were achieved by fitting a 2D Gaussian distribution of fixed width on top of an inclined plane representing the sky background and stray light from nearby stars. The fixed width of the Gaussian profile was derived from bright nonsaturated field stars and the photometric scale measured on averaged images since the flux from bright stars is not accurately conserved in the image stacking process. Colour transformations provided by ESO were systematically applied in order to compensate for the large colour differences between RX J0720.4-3125 and the photometric field reference stars. Magnitudes derived from the various runs are listed in Table 3. The 1999-2003 B magnitude of RX J0720.4-3125 is consistent with that reported by Kulkarni $\&$ van Kerkwijk (1998), $B=26.6 \pm 0.2$, and the re-analysis of the 1997 images using 2D Gaussian fitting instead of aperture photometry also provides consistent values, $B=26.58 \pm 0.25$.
Table 3. $U$ and $B$ magnitudes of RX J0720.4-3125 ( $1 \sigma$ errors).

\begin{tabular}{ccc}
\hline \hline Epoch & $U$ & $B$ \\
\hline February-April 1997 & & $26.58 \pm 0.25$ \\
January 1999 & & $26.79 \pm 0.20$ \\
January 2000 & $25.68 \pm 0.17$ & $26.44 \pm 0.15$ \\
December 2000 & & $26.787 \pm 0.040$ \\
Dec. 2002-Jan. 2003 & $26.620 \pm 0.050$ \\
\hline
\end{tabular}

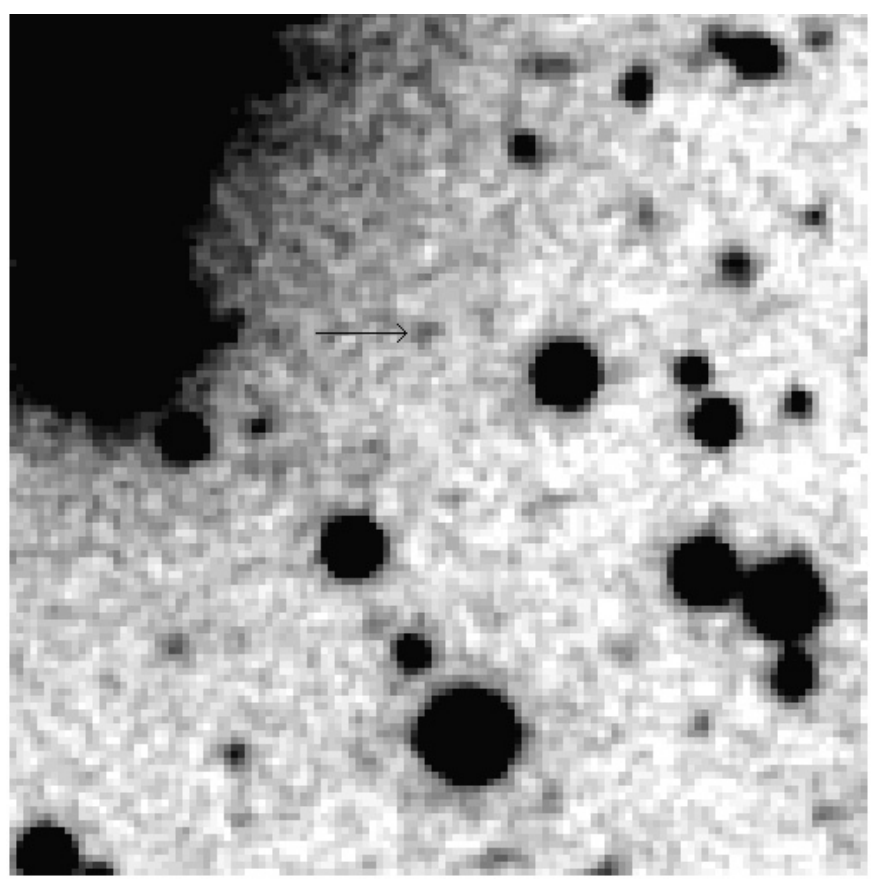

Fig. 2. The summed $U$ band image obtained with EFOSC2 on the ESO-3.6 $\mathrm{m}$ in January 2000. The area covered is roughly the same as that seen in Fig. 1. The arrow shows the position of RX J0720.4-3125.

The $U-B$ index is very blue, $U-B=-1.11 \pm 0.18$, assuming no $B$ optical flux variation. The simultaneous but less accurate $B$ measurement in January 2000 yields a consistent $U-B=-0.76 \pm 0.23$. Our estimate of the $U$ band flux agrees well with the optical to far UV distribution measured by Kaplan et al. (2003). The difference of $0.17 \mathrm{mag}$ in $B$ between the two ESO-VLT observations is only a $2.6 \sigma$ effect and is thus probably not significant. We also used the high quality optical data from the last ESO-VLT run to search for night to night variability. There again, we find some scatter with a maximum amplitude of $0.25 \mathrm{mag}$ from one night to the other which is only significant to a $2.9 \sigma$ level. It is more likely that the rather large photometric scatter is due to a slight underestimate of the actual photometric errors.

\section{Proper motion study}

We searched for possible proper motion by comparing the positions of the optical counterpart at five epochs from 1997 to 2003. Apart from the 1997 images which are the sum of data collected over a time interval of 2 months from February 7 to April 4, the four other epoch observations took place 
Table 4. Maximum errors (milli-arcseconds) in RA and Dec for the astrometric reference stars.

\begin{tabular}{ccc}
\hline \hline Epoch & RA & Dec \\
\hline Feb.-Apr. 1997 & 12.0 & 4.2 \\
January 1999 & 4.4 & 4.3 \\
January 2000 & 32 & 34 \\
December 2000 & 4.1 & 7.0 \\
Dec. 2002 Jan. 2003 & 5.2 & 3.2 \\
\hline
\end{tabular}

during integer numbers of consecutive years within 27 days from the same calendar date. Overall, the change in position due to trigonometric parallax is expected to be of the order of 16 mas at maximum for an assumed distance of $100 \mathrm{pc}$ (mainly due to the spread in time of the 1997 observations), and can therefore be neglected for our purpose. As for the photometry we found that fitting 2D Gaussian profiles gave the most accurate results and good estimates of the positional errors. Positions from automated processes such as Sextractor or DAOPHOT turned out to be less reliable in general. The fixed width of the stellar profiles in right ascension and declination were first derived by averaging the values obtained on a set of relatively bright but nonsaturated stars. Then the position of a group of 10 reference stars was measured, together with that of RX J0720.4-3125 and of 17 faint test objects. Reference stars are evenly spread within 30" of RX J0720.4-3125 and are in the range of $B$ magnitude from 21.8 to 18.8 . Comparison objects are distributed in the same sky region as the reference stars and were selected for their star-like appearance. Their magnitudes range from $B \sim 27.0$ to 23.5 . Using a rotation, translation and scaling transformation, the 1997, 1999, 2000 January and 2003 positions were then moved to the 2000 December FORS1/VLT reference frame which provides the most accurate positions. Not all the reference stars are common to all frames and the 1997 to December 2000 transformation was computed with only 8 stars. We checked that the quality of the transformation was not depending heavily on the number of astrometric reference stars used. Since part of the proper motion could be due to bulk motion of the reference stars we also computed the astrometric solution of the December 2000 VLT image using 78 USNO-A2 field stars. Reference stars do not show any systematic difference in position in the time interval from 1980 (USNO-A2) till 2000. The accuracy with which reference stars coincide after transformation is rather good. Their maximum offset to mean positions after transformation are listed in Table 4. Between December 2000 and 2002-2003 VLT observations, the mean residual of the 10 reference stars is only 4.5 mas. We plot in Fig. 3 the apparent displacements of the 17 faint comparison stars together with those of RX J0720.4-3125 and of the astrometric references derived from the two FORS1/VLT observations. None of the reference stars exhibit significant motion with respect to the rest of the group. Compared to stars of similar brightness, the apparent motion of RX J0720.4-3125 is clearly of high significance.

We also corrected for differential refraction effects resulting from the large colour difference between RX J0720.4-3125

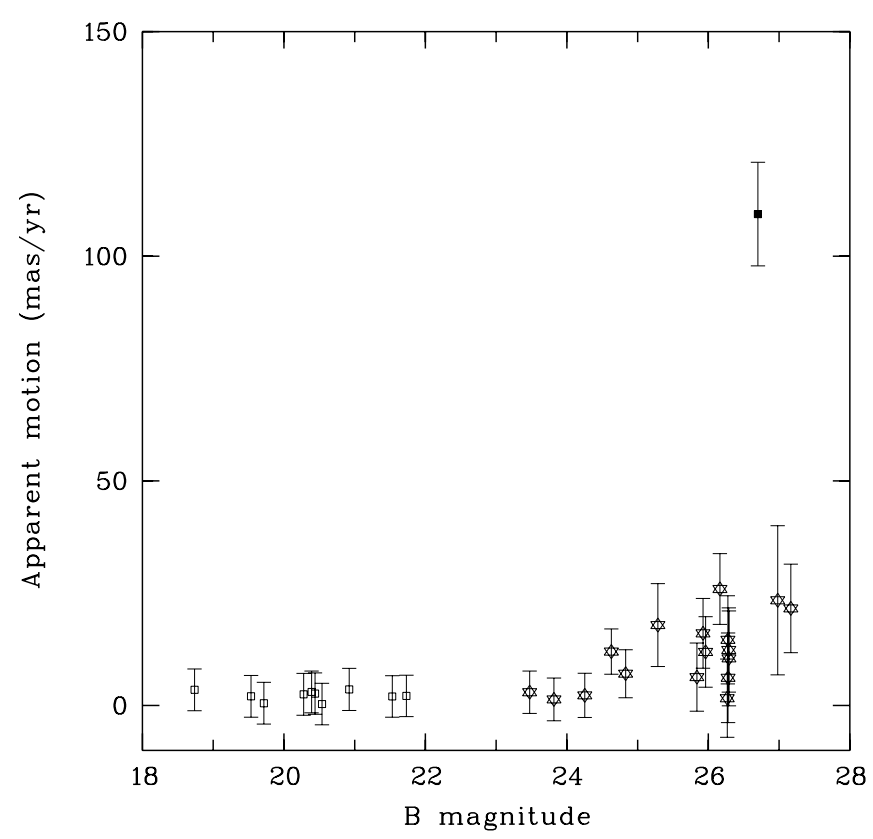

Fig. 3. Apparent proper motion of local astrometric reference stars (open squares), comparison objects (stars) and of RX J0720.4-3125 (filled square) using December 2000 and December 2002January 2003 VLT images.

$(U-B=-1.11)$ and the reference astrometric stars (mean $U-B=0.17)$. In the $B$ filter, this translates into a shift of $\sim 130 \AA$ in effective wavelength. Since all observations were done at low airmass, the corrections remain small, of the order of 5 to 30 mas and are usually negligible compared to positioning errors. Differential refraction effects are only significant in the case of the December 2000 VLT observation.

We then fitted a proper motion solution expressed in terms of total motion and direction angle to the various positions of RX J0720.4-3125 using a minimum $\chi^{2}$ algorithm. The best fit shown in Fig. 4 is obtained for $\mu=97 \pm 12$ mas/yr. The direction of motion is oriented $29^{\circ} \pm 7^{\circ}$ north of west (all errors are $90 \%$ confidence level for one parameter).

\section{Optical to X-ray energy distribution}

In the X-ray domain RX J0720.4-3125 was observed with the ROSAT, SAX, XMM-Newton and Chandra observatories. As results inferred from different instruments are subject to calibration uncertainties (see, e.g., Burwitz et al. 2001 for the case of RX J1856.5-3754), we used only the Chandra data collected with the LETGS grating instrument in a total exposure of $37.5 \mathrm{ks}$ in February 2000. First results from the spectral analysis of these data have been presented in Pavlov et al. (2002). They found that neither light-element (hydrogen or helium) nor heavy-element (pure iron or solar mixture) neutron star atmosphere models can explain the multi-wavelength spectral distribution of the emission detected from RX J0720.4-3125. The net result of this analysis was remarkably similar to the situation with the spectral fitting of the optical and X-ray data on RXJ1856.5-3754: while providing acceptable fits to the $\mathrm{X}$-ray data the light-element atmosphere models yield unrealistic large radii of the INS and significantly over-predict the 


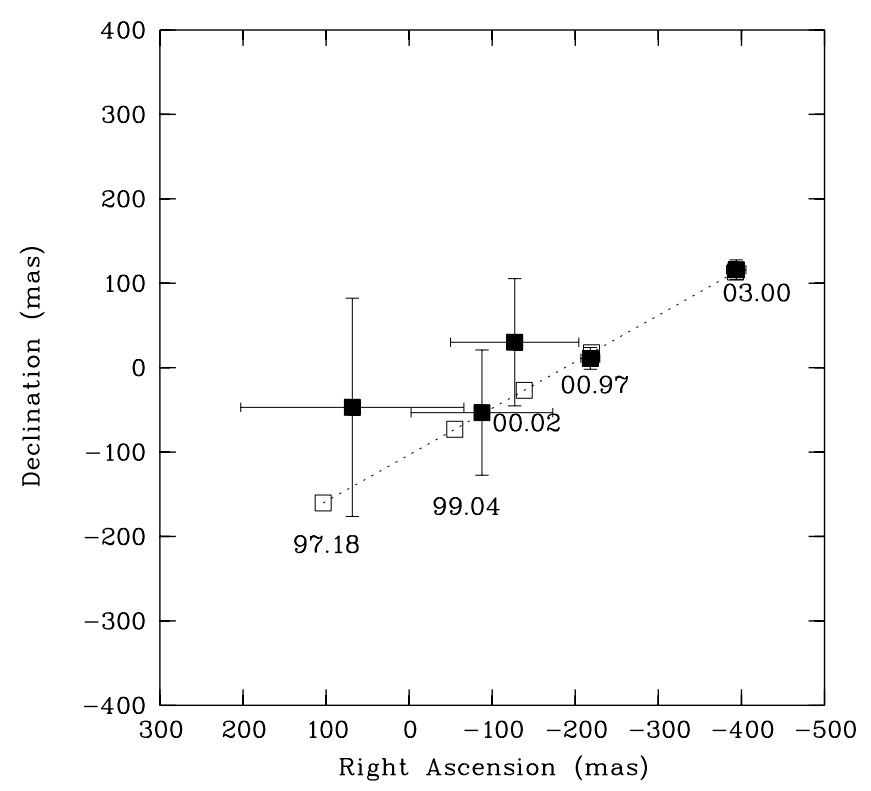

Fig. 4. Positions of RXJ0720.4-3125 for the five epochs (filled squares) together with the best fit proper motion solution (open squares) corresponding to $\mu=97$ mas/yr.

actual optical fluxes (by a factor of $\sim 300$ ), whereas the heavyelement models do not fit the X-ray data due to numerous features in the model spectra caused by absorption at atomic transitions in ions of the heavy elements (see Zavlin \& Pavlov 2002 for details). Pavlov et al. (2002) found that the best fit to the Chandra LETGS data on RX J0720.4-3125 is provided by a blackbody model with the temperature $k T_{\mathrm{bb}}^{\infty}=80-82 \mathrm{eV}$ emitted from an area of a radius $R_{\mathrm{bb}}^{\infty}=(2.0-2.2)(D / 100 \mathrm{pc}) \mathrm{km}$ ( $D$ is the distance to the source), absorbed with the hydrogen column density $N_{\mathrm{H}}=(1.5-1.7) \times 10^{20} \mathrm{~cm}^{-2}$.

Similar to the case of RX J1856.5-3754, the best blackbody model derived from the X-ray data on RX J0720.4-3125 underpredicts the optical fluxes detected from the source by a factor of 4. Pavlov et al. (2002) speculated that a two-blackbody model, originally suggested by Pons et al. (2002) to describe the multi-wavelength data on RX J1856.5-3754, may be also applied to the radiation from RX J0720.4-3125, with the "soft" component of $k T_{\mathrm{bb}, \mathrm{s}}^{\infty}<43 \mathrm{eV}$ and $R_{\mathrm{bb}, \mathrm{s}}^{\infty}>6.1(D / 100 \mathrm{pc}) \mathrm{km}$. Such a model, with $\mathrm{X}$-rays originating from a hot area on the star's surface and the optical fluxes emitted from the rest of the surface, may explain the pulsations of the X-ray radiation from RX J0720.4-3125. In addition to the thermal components, HST observations by Kaplan et al. (2003) reveal evidence for a nonthermal power law component.

However, while this simple multiple-component model seems to be in agreement with the properties of the emission detected from RX J0720.4-3125, it is hardly reconciled with the fact that, as stars are not blackbodies, radiation emitted by a star should deviate from a blackbody model. Also, in the case of RX J1856.5-3754, the absence of pulsations put severe constraints on the two-blackbody model, requiring either a particular geometrical configuration or strong gravitational deflection (Ransom et al. 2002).
All previous models of neutron star surface radiation were based on a conventional assumption that there is enough matter (e.g., pure hydrogen or iron, or a mixture of elements) on the neutron star surface to make the atmosphere layers optically thick at all energies of interest. A typical estimate for such an amount in terms of the total surface column density is $y_{\text {tot }}>10-100 \mathrm{~g} \mathrm{~cm}^{-2}$ (depending on the surface temperature) to provide the equilibrium (or diffusion) solution of the radiative transfer problem in the very deep layers (see Mihalas 1978). Under this assumption, spectra of the emitted radiation are solely determined by the temperature distribution in the atmosphere (which grows towards larger depths) and do not depend on properties of star's layers lying underneath the atmosphere. In the case of a light-element atmosphere composition, the model spectra were found to be much harder at higher photon energies than blackbody ones providing the same radiative flux. The reason for this effect is that high-energy photons with longer mean-free-paths are emitted from deep surface layers with temperatures larger than the so-called effective temperature, the fourth power of which determines the total energy flux (see, e.g., Zavlin et al. 1996; Zavlin \& Pavlov 2002, for details). Due to this property the model spectra, when applied to observational data, usually yield lower temperatures by a factor of 2-3 and much larger emitting areas by a factor of 50-200 than estimates obtained from blackbody fits. However, it is not known a priori how much, for example, hydrogen has been deposited on the surface of a neutron star (e.g., by accretion from the interstellar medium or from a fallback disk). If the hydrogen layer is not optically thick at all energies, but only at lower ones, then the atmosphere structure (temperature run) is modified, that in turn should affect the spectra of emergent radiation.

We investigated nonmagnetic atmosphere models with various values of the total column density of hydrogen $y$ tot in atmosphere. We assumed the atmosphere to be in radiative and hydrostatic equilibrium, and that the radiation at the inner boundary $y=y_{\text {tot }}$ is given by the diffusion solution (although the latter condition is not strictly justified - see Discussion). The main result we obtain is rather natural: reducing the total column density lowers the temperature of surface layers that emit high-energy photons, that in turn leads to the softening of the Wien tail in the model spectra, making them close to the blackbody ones with the same effective temperature. This means that optical fluxes predicted by these models applied to $\mathrm{X}$-ray data on INSs are expected to be significantly decreased in comparison with those given by the standard model, but still larger than values yielded by the blackbody model. We computed hydrogen models in a grid of effective temperature and total column density (down to $y_{\text {tot }}=10^{-3} \mathrm{~g} \mathrm{~cm}^{-2}$ ) and applied these models to the UV - optical and Chandra LETGS data on RX J0720.4-3125 taking into account the nonthermal power law discovered by Kaplan et al. (2003). A best fitting model is shown in Fig. 5. The parameters of the model are: total atmosphere column density $y_{\text {tot }}=0.16 \mathrm{~g} \mathrm{~cm}^{-2}$, effective temperature $k T_{\text {eff }}=57 \mathrm{eV}$, distance to the star $D=204 \mathrm{pc}$ (assuming the standard neutron star radius $R=10 \mathrm{~km}$ and mass $M=1.4 M_{\odot}$ ), and interstellar absorption $N_{\mathrm{H}}=1.1 \times 10^{20} \mathrm{~cm}^{-2}$. As seen in Fig. 5, the model nicely fits the LETGS data $\left(\chi_{v}^{2}=0.94\right.$ in the X-ray domain) and agrees well with the detected optical 


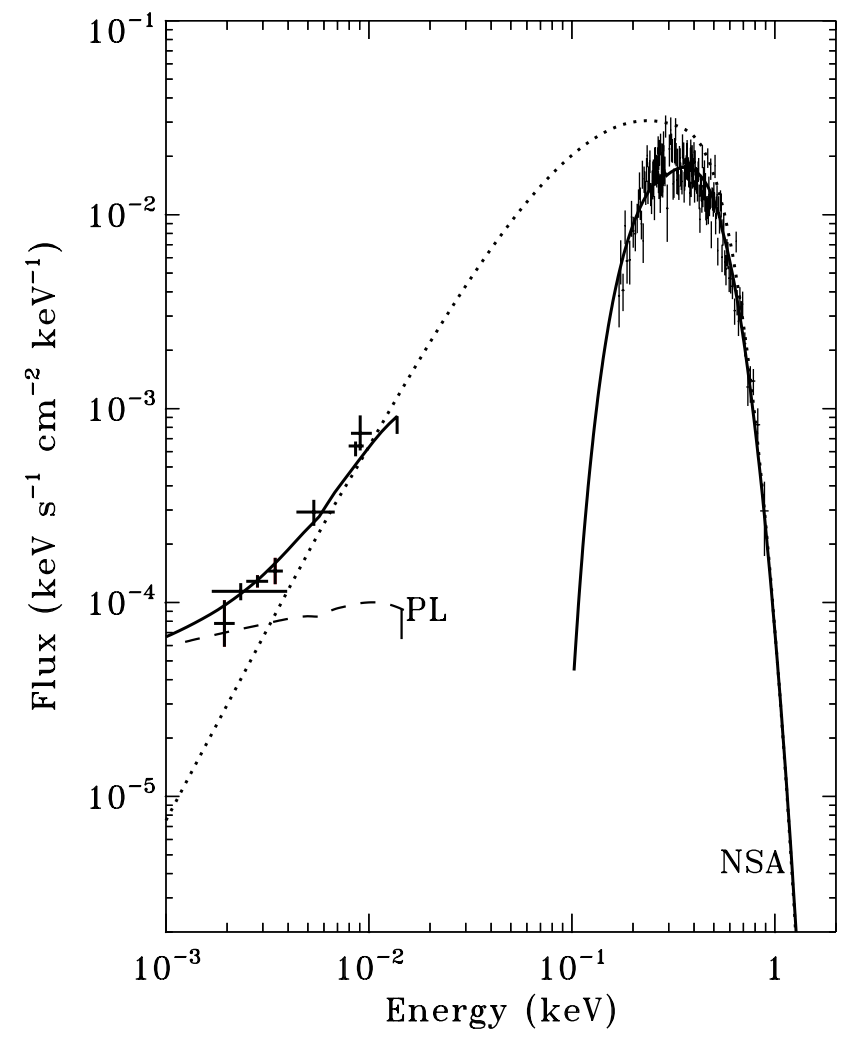

Fig. 5. Multiwavelength energy distribution of RX J0720.4-3125. The optical and far UV data are from Kulkarni \& van Kerkwijk (1998), Kaplan et al. (2003) and this paper. The X-ray spectrum is that from the Chandra LEGTS data. Solid line shows the best thin neutron star atmosphere (NSA) + nonthermal power law (Kaplan et al. 2003, dashed line) model fitting both the X-ray and optical data (see text for the parameters). The dotted line gives unabsorbed fluxes.

fluxes. The total mass of hydrogen obtained in the fit is $4 \pi R^{2}$ $y_{\text {tot }}=2 \times 10^{12} \mathrm{~g}$. Assuming a stellar age of $10^{6} \mathrm{yr}$, this translates into a mean accretion rate of $\sim 0.6 \mathrm{~g} / \mathrm{s}$, which is many orders of magnitude lower than predicted by the accretion model.

\section{Discussion}

Despite the fact that the thin atmosphere model provides a good fit to the observational data in the broad energy range at reasonable neutron star parameters, it serves rather as an illustrative example because of a number of shortcomings in the simplified approach it has been derived. First of all, the assumption with which the equilibrium solution at the maximal depth in such an atmosphere may not be justified at higher X-ray energies where the emitted radiation could be affected by properties of the stellar surface under the atmospheric layer. It means that the boundary condition at the inner boundary $y=y_{\text {tot }}$, taken currently to be the same as in the standard modeling (with much larger values of $y_{\text {tot }}$ ), may need to be modified. For instance, one could consider such a thin atmosphere on top of a solid neutron star surface or a thicker layer composed of another chemical element. Second, the model should be developed with account for the presence of a strong surface magnetic field, $B \sim 10^{13} \mathrm{G}$, as recently suggested by Zane et al. (2002). It is safe to assume that in the case of magnetized models, reducing the atmosphere thickness would also soften the spectrum of the emergent radiation. Since in strong magnetic fields the emergent spectra of the standard models are intrinsically softer than the non-magnetic ones (Zavlin \& Pavlov 2002), one may expect that the magnetized model needs a larger atmosphere thickness in order to describe the broad-band properties of the emission from RX J0720.4-3125.

Another point here is that this model is constructed assuming a uniform neutron star surface, which makes the model emission unpulsed, whereas the observed X-rays from RX J0720.4-3125 reveal pulsations with a pulsed fraction of about $10 \%$. A way to model the pulsations is to consider a nonuniform temperature distribution over the surface (see, e.g., Cropper et al. 2001) since such a temperature nonuniformity can be induced by the strong magnetic field (Greenstein \& Hartke 1983). Finally, in its present state, the thin atmosphere model applied to the multiwavelength data on RX J1856.5-3754 underpredicts the distance to this object by a factor of 2. In light of all this, the presented spectrum should be considered as a first, perhaps rather crude step towards more advanced modeling of radiation from a neutron star surface covered with a thin atmosphere layer, which might help to elucidate the origin of thermal emission detected from INSs.

A proper motion of $97 \pm 12$ mas per year corresponds to a transverse velocity of $V_{\mathrm{T}} \sim 50 \times(D / 100 \mathrm{pc}) \mathrm{km} \mathrm{s}^{-1}$. A small part of this displacement could be due to solar motion (about $12 \mathrm{mas} / \mathrm{yr}$ in the right ascension if the source is at a distance of $100 \mathrm{pc}$ ). RX J0720.4-3125 is thus the second radio-quiet soft X-ray bright isolated neutron star for which proper motion is detected. The value reported here is about a third of that measured for RX J1856.5-3754 (Neuhäuser 2001; Walter 2001; Kaplan et al. 2002).

Such a spatial motion, although modest for neutron star standards, most probably rules out the accretion scenario for RX J0720.4-3125. The observed bolometric luminosity of $2.6 \times 10^{31}(D / 100 \mathrm{pc})^{2} \mathrm{erg} \mathrm{s}^{-1}$ (Haberl et al. 1997) implies accretion rates of the order of $\dot{M} \sim 1.2 \times 10^{11}(D / 100 \mathrm{pc})^{2} \mathrm{~g} \mathrm{~s}^{-1}$. On the other hand, the accretion rate can be estimated assuming the Bondi-Hoyle model: $\dot{M} \sim n v_{10}^{-3} \times 10^{11} \mathrm{~g} \mathrm{~s}^{-1}$ where $n$ is the mean density of the interstellar medium (in $\mathrm{cm}^{-3}$ ) and $v_{10}$ the relative velocity in units of $10 \mathrm{~km} \mathrm{~s}^{-1}$ (Treves et al. 2001). Therefore, very high ISM densities of the order of $n \sim$ $150(D / 100 \mathrm{pc}) \mathrm{cm}^{-3}$ are required to account for the observed $\mathrm{X}$-ray flux in the accretion scenario. This density is much higher than the mean density derived from soft X-ray photoelectric absorption or from optical reddening of field stars, $n \sim$ $0.2-0.4 \mathrm{~cm}^{-3}$ (Motch \& Haberl 1998). However, there have been recent reports on evidence for dense ISM structures $(n \sim$ $10-1000 \mathrm{~cm}^{-3}$ ) on small scales ranging from 10 to $10^{6} \mathrm{AU}$ in regions of otherwise low mean densities (Lauroesch \& Meyer 2000 and Meyer \& Lauroesch 1999). RX J0720.4-3125 could be presently passing through one of these high density structures at a velocity of $\geq 10 \times(D / 100 \mathrm{pc}) \mathrm{AU} \mathrm{yr}^{-1}$. Since such high density clouds are likely to be rare, we would only detect X-ray emission from old neutron stars when they pass through these small regions. The X-ray ionization of these cloud-lets may be detectable in the optical. It is not clear, however, whether the apparent absence of X-ray flux and 
temperature changes from RX J0720.4-3125 is compatible with the size of these dense structures and the expected time scale for accretion instability. This scenario furthermore, cannot account for the large $\dot{P} \geq 10^{-14} \mathrm{~s} \mathrm{~s}^{-1}$ measured by Zane et al. (2002).

Another implication of the high proper motion is that in no case can the spin-down derived by Zane et al. (2002) be due to a propeller torque extracted from the interstellar medium. Following Colpi et al. (1998), propeller spin-down can be written as $\dot{P} \sim 3.1 \times 10^{-16} n^{9 / 13} v_{10}^{-27 / 13} B_{12}^{8 / 13} P^{21 / 13} \mathrm{~s} \mathrm{~s}^{-1}$ where $B_{12}$ is the polar magnetic field in units of $10^{12} \mathrm{G}$. At $\dot{P}=$ $5.4 \times 10^{-14} \mathrm{~s} \mathrm{~s}^{-1}$ the propeller mechanism dominates over the magnetic dipole losses if $v_{10} \leq 0.9 n^{1 / 3}$. Since $v_{10}$ is likely greater than unity, the propeller mechanism could only dominate if the star presently goes through a high density medium with $n \geq 100$.

The possibility that RX J0720.4-3125 breaks through propeller interaction with a fallback disk seems as well unlikely. First, assuming that the entire $X$-ray luminosity is due to friction leads to much larger $\dot{P}$ than observed (Zane et al. 2002; Kaplan et al. 2002).

In the framework of the blackbody interpretation of the X-ray data, a part of the optical flux excess above the extrapolation of this model to the low-energy band (see Sect. 5 and Fig. 5) could arise from the reprocessing of X-rays in a residual debris disk when the neutron star has entered the pulsar phase. Emission spectra of such discs were computed by Perna et al. (2000) in a number of configurations. Although in the $B$ band, the entire flux excess above the X-ray blackbody extrapolation could be due to a remnant disc, its energy distribution is rather red and would fail to account for the $U$ band excess. Therefore, it is not clear whether such discs can significantly contribute to the overall optical emission of RX J0720.4-3125. Similar conclusions are reached by Kaplan et al. (2003).

The lack of parallax and radial velocity as well as the large uncertainty on the parameters of the proper motion do not allow one to guess the birth place of RX J0720.4-3125 as it is apparently possible for RX J1856.5-3754 (Walter \& Lattimer 2002). The star is moving away from the Galactic plane but is still very close to it. Its general direction of motion is consistent with a birth either in the Sco OB2 complex, as for RX J1856.5-3754 (more probably in the lower Centaurus Crux region at $D \sim 170 \mathrm{pc}$ ), or in the Vela OB2 + Trumpler 10 association $(D=360-410 \mathrm{pc})$. In both cases, the flight time from birth place is of the order of $10^{6} \mathrm{yr}$ with a rather large uncertainty. This value is generally consistent with the spin down age and with cooling time (see Zane et al. 2002).

Several high magnetic field $\left(B \geq 10^{13} \mathrm{G}\right)$ radio pulsars with long periods have been recently discovered (Camilo et al. 2000; Morris et al. 2002). One of these radio pulsars, PSR J1830-1135 has properties astonishingly close to those of RXJ0720.4-3125 $\left(P=6.22 \mathrm{~s}, \dot{P}=4.7 \times 10^{-14} \mathrm{~s} \mathrm{~s}^{-1}\right.$ and $B=1.7 \times 10^{13} \mathrm{G}$, Morris et al. 2002). It could thus well be that RX J0720.4-3125 and several other X-ray bright and radio-quiet INSs are radio pulsars whose radio beams do not cross the Earth (Motch 2001; Kaplan et al. 2002, 2003). The effect of radio beam narrowing with increasing periods (Biggs 1990) could plausibly explain the much smaller ratio of long-period to short-period radio pulsars compared to that found for X-ray pulsars.

\section{Conclusions}

ESO-VLT and $4 \mathrm{~m}$ class telescope imaging reveal that RX J0720.4-3125 has a proper motion of $\mu=97 \pm 12$ mas/yr. Such a relatively high spatial velocity almost completely rules out the possibility that the neutron star accretes matter from the interstellar medium. This strongly favours the idea that RX J0720.4-3125 is a cooling INS of an age around $10^{6} \mathrm{yr}$. A propeller mechanism on a fallback disk or on accreted material from the ISM cannot account for the braking detected by Zane et al. (2002) and thus the observed spin-down is likely due to magnetic dipole losses. Recent discoveries of radio pulsars with comparable periods and magnetic fields combined with the effect of the radio beam narrowing at longer periods suggest that RX J0720.4-3125 could well be an off-beam radio pulsar. As in RX J1856.5-3754, the optical continuum lies well above the extrapolation of the blackbody model seen in X-rays. To account for the optical emission one can add a lower temperature blackbody component cold enough not to be detected in the X-ray range (Pavlov et al. 2002; Kaplan et al. 2003). However, neutron stars are unlikely to radiate like blackbodies. Spectra predicted by heavy-element atmosphere models have a number of prominent features which should be conspicuous in the Chandra and XMM-Newton data obtained with high-energy resolution. On the other hand, the standard (thick) hydrogen atmosphere models overpredict the optical emission by several magnitudes. We propose here a new kind of model atmosphere in which the thickness of the light-element layer has reduced values. Although the model still has a number of shortcomings, it can adequately fit the overall energy distribution with a single component, requiring the distance to RX J0720.4-3125 to be about $200 \mathrm{pc}$ which still has to be compared with the actual value to be provided by future parallax measurements.

Acknowledgements. We thank the referee, Patrick Slane, for very valuable comments. VEZ acknowledges discussions on the atmosphere model modifications with Bernd Aschenbach, George Pavlov and Joachim Trümper.

\section{References}

Biggs, J. D. 1990, MNRAS, 245, 514

Blaes, O., \& Madau, P. 1993, ApJ, 403, 690

Burwitz, V., Zavlin, V. E., Neuhäuser, R., et al. 2001, A\&A, 379, 35

Burwitz, V., Haberl, F., Neuhäuser, R., et al. 2003, A\&A, 399, 1109

Camilo, F., Kaspi, V. M., Lyne, A. G., et al. 2000, ApJ, 541, 367

Colpi, M., Turolla, R., Zane, S., \& Treves, A. 1998, ApJ, 501, 252

Cropper, M., Zane, S., Ramsay, G., Haberl, F., \& Motch, C. 2001, A\&A, 365, L302

Drake, J. J., Marshall, H. L., Dreizler, S., et al. 2002, ApJ, 572, 996

Greenstein, G., \& Hartke, G. J. 1983, ApJ, 271, 283

Haberl, F. 2003, in High Energy Studies of Supernova Remnants and Neutron Stars, ed. W. Becker, \& W. Hermsen, COSPAR Symposium, Houston 2002, in press [astro-ph/0302540]

Haberl, F., Motch, C., Buckley, D. A. H., Zickgraf, F. J., \& Pietsch, W. 1997, A\&A, 326, 662 
Kaplan, D. L., Kulkarni, S. R., van Kerkwijk, M. H., \& Marshall, H. L. 2002, ApJ, 570, 79

Kaplan, D. L., van Kerkwijk, M. H., \& Anderson, J. 2002, ApJ, 571, 447

Kaplan, D. L., van Kerkwijk, M. H., Marshall, H. L., Jacoby, B. A., \& Kulkarni, S. R. 2003, ApJ, in press

Kulkarni, S. R., \& van Kerkwijk, M. H. 1998, ApJ, 507, L49

Landolt, A. U. 1992, AJ, 104, 340

Lauroesch, T. J., \& Meyer, D. M. 2000, ApJ, 543, L43

Madau, P., \& Blaes, O. 1994, ApJ, 423, 748

Meyer, D. M., \& Lauroesch, T. J. 1999, ApJ, 520, L103

Mihalas, D. 1978, Stellar Atmospheres (San Francisco: Freeman)

Morris, D. J., Hobbs, G., Lyne, A. G., et al. 2002, MNRAS, 335, 275

Motch, C., \& Haberl, F. 1998, A\&A, 333, L59

Motch, C. 2001, in AIP Conf. Proc., ed. N. E. White, G. Malagutti, \& G. G. C. Palumbo, 599, 244

Neuhäuser, R. 2001, AN, 322, 3

Paerels, F., Mori, K., Motch, C., et al. 2001, A\&A, 365, 298
Pavlov, G. G., Zavlin, V. E., Trúmper, J., \& Neuhäuser, R. 1996, ApJ, 472, L33

Pavlov, G. G., Zavlin, V. E., \& Sanwal, D. 2002, in Neutron Stars, Pulsars and Supernova, ed. W. Becker, H. Lesch, \& J. Trümper, MPE Report 278, 273 [astro-ph/0206024]

Perna, R., Hernquist, L., \& Narayan, R. 2000, ApJ, 541, 350

Pons, J. A., Walter, F. M., Lattimer, et al. 2002, ApJ, 564, 981

Ransom, S. M., Gaensler, B. M., \& Slane, P. O. 2002, ApJ, 570, L75

Treves, A., Turolla, R., Zane, S., \& Colpi, M. 2000, PASP, 112, 297

Walter, F. M. 2001, ApJ, 549, 433

Wang, J. C. L., ApJ, 486, L119

Walter, F. M., \& Lattimer, J. M. 2002, ApJ, 576, L45

Zane, S., Haberl, F., Cropper, et al. 2002, MNRAS, 334, 345

Zavlin, V. E., Pavlov, G. G., \& Shibanov, Yu. A. 1996, A\&A, 315, 141

Zavlin, V. E., \& Pavlov, G. G. 2002, in Neutron Stars, Pulsars and Supernova, ed. W. Becker, H. Lesch, \& J. Trümper, MPE Report, 278, 263 [astro-ph/0206025] 